\title{
SIMPLY CONNECTED MANIFOLDS WITH NO CONJUGATE POINTS WHICH ARE FLAT OUTSIDE A COMPACT SET
}

\author{
CHRISTOPHER B. CROKE
}

(Communicated by Jonathan M. Rosenberg)

\begin{abstract}
In this note we improve a previous result to show that euclidean $n$ space $(n \geq 3)$ is the only simply connected manifold without conjugate points which is flat outside a compact set.
\end{abstract}

Our goal is to prove the following:

Theorem. If $M$ is a complete, simply connected Riemannian manifold of dimension $n \geq 3$ which has no conjugate points and is flat outside a compact set, then $M$ is isometric to $\mathbb{R}^{n}$.

This is an improvement of Theorem A of [C], where we prove the same theorem under the assumption that the complement of a compact set is isometric to the complement of a compact set in $\mathbb{R}^{n}$. The proof here consists of reducing the above theorem to Theorem A of [C].

The theorem is clearly false for $n=2$. However, as had been shown earlier in [G-G], Theorem A of [C] does hold in this case as well.

Proof. Pick $x \in M$, and choose $R$ so large that $M-B(x, R / 2)$ is flat, where $B(x, R)$ represents the ball of radius $R$ about $x$. Let $S(x, R)$ be the boundary of $B(x, R)$. Since $M$ has no conjugate points and is simply connected, $M$ is diffeomorphic to $\mathbb{R}^{n}, B(x, R)$ is diffeomorphic to an $n$-ball, and $S(x, R)$ is diffeomorphic to an $n-1$ sphere.

We claim that the second fundamental form of $S(x, R)$ is definite. Let $y \in S(x, R)$ and $\gamma$ be the geodesic with $\gamma(0)=x$, and $\gamma(R)=y$. Let $e$ be an eigenvalue of the second fundamental form of $S(x, R)$ at $y$, and let $X \in T_{y} S(x, R)$ be a corresponding eigenvector. If $\tau(s) \in S(x, R)$ is a curve with $\tau^{\prime}(0)=X$, then the variation of geodesics $H(s, t)=\operatorname{Exp}_{\tau(s)}(t-R) N(\tau(s))$, where $N(\tau(s))$ is the outward normal to $S(x, R)$ at $\tau(s)$, has as variation field the Jacobi field $J(t)$ with $J(R)=X$ and $J^{\prime}(R)=e X$. Further, since $H(s, 0)=x, J(0)=0$. Since there are no conjugate points, $J$ cannot vanish for $t \neq 0$ and, by [E-O], must get arbitrarily large as $t \rightarrow \infty$. For $t \geq R, M$

Received by the editors September 22, 1989.

1980 Mathematics Subject Classification (1985 Revision). Primary 53C20; Secondary 53C25.

Research supported by NSF grant DMS87-22998 and M.S.R.I. 
is flat at $\gamma(t)$, and hence $J(t)=(1+e \cdot t) \bar{X}$, where $\bar{X}$ is the parallel field along $\gamma$ with $\bar{X}(R)=X$. Thus $e>0$.

The rest of the argument proceeds as in Lemma 1 of [S-S]. Since $n \geq 3$, $M-B(x, R / 2)$ is simply connected and hence admits an isometric immersion (the development map) $F$ into $\mathbb{R}^{n}$. Since the second fundamental form of $S(x, R)$ is definite, $F(S(x, R))$ must be an embedded convex surface [S]. Since $F$ takes geodesics normal to $S(x, R)$ to geodesics normal to $F(S(x, R))$, it is easy to see that $F$ is an isometry from $M-B(x, R)$ to the unbounded component of $\mathbb{R}^{n}-F(S(X, R))$.

The theorem now follows from Theorem $\mathrm{A}$ of $[\mathrm{C}]$.

\section{REFERENCES}

[C] C. Croke, Rigidity and the distance between boundary points, J. Differential Geom. (to appear).

[E-O] J. Eschenberg and J. O'Sullivan, Growth of Jacobi fields and divergence of geodesics, Math. Z. 150 (1976), 221-237.

[G-G] L. Green and R. Gulliver, Planes without conjugate points, J. Differential Geom. 22 (1985), 43-47.

[S-S] V. Schroeder and M. Strake, Rigidity of convex domains in manifolds with non-negative Ricci and sectional curvature, Comment. Math. Helv. 64 (1989), 173-186.

[S] R. Sacksteder, On hypersurfaces with nonnegative sectional curvature, Amer. J. Math. 82 (1982), 609-630.

Department of Mathematics, University of Pennsylvania, Philadelphia, PennsylvaNIA 19104 